\title{
Editorial
}

\section{A renaissance for the 'sense of wonder'?}

\author{
Martyn Evans, David Greaves Centre for Philosophy and Health Care, University of Wales
}

Medical humanities as an aspiration looks both forward and backward, seeking in part the rediscovery of a certain attitude towards medicine, its natural objects/subjects (patients) and its place in the cultural, artistic and scientific order. That attitude is embodied in the idea of a Renaissance Man or Woman embracing an interdisciplinary (even omnidisciplinary) view of the world, a universal gaze which is, on the face of it, no longer available to us in a world of run-away specialisation in knowledge. Consider how one might both celebrate "the beauty of the human form and the nobility of the human spirit" and pursue "an insatiable curiosity for the materiality of the here and now, a Faustian itch to explore ..."1-a universal gaze which, fused within an individual mind, may seem paradoxical, rather than merely daunting.

Be that as daunting - or as paradoxical-as it may, we suggest that one source of both moral and intellectual renaissance for the contemporary physician lies in recapturing a sense of wonder at the human body, its place in the natural realm, and its miraculous functioning as the fount, and the medium, of embodied human experience. Such a sense of wonder has epistemological, moral and spiritual dimensions; all are reflected in this issue's first paper, reviewing literary responses to the physical deconstruction (and the attempted demystification) of the human body in medical school dissection. ${ }^{2}$ The paper's authors, one general practitioner and a teacher and another a medical student, trace the historical erosion of a sense of wonder and respect for the human body in the study of dissection.

Regrettable in itself, the erosion of that sense of wonder also leads to a sense of the body's mere instrumentality - as it were, its "usefulness" both to the person who is, or was, that body and to those who might make use of the body subsequently. And this engages the medical humanities with a very contemporary moral issue, the large-scale removal and storage of organs from children who have died in British hospitals. ${ }^{3}$ One might ask: Isn't this exactly what can go wrong when a sense of wonder for the human body is lost?
Initially the answer seems to be a simple "yes". At any rate we need a widened notion of respect in order to interpret the popular upset and outrage concerning these organ removal practices - and the government's projected response (primarily, laws to protect relatives' control over of the bodies their deceased loved ones). But at a deeper level this response seems incomplete, a merely mechanical view of both the problem and its solution: as if the shortcomings concerned merely obtaining permission regarding the use of someone's "property", and their solution simply required compliance with procedures of institutional practice-the reascription of "property rights", away from hospitals and back to patients or relatives.

Absent is any change in attitude towards a more communal sense of what it is that clinicians and pathologists are dealing with-the mortal, experiential fabric of human beings. Whatever the motivation of any individual pathologist, the collective practice of pathologists at least attempted to serve our understanding of patients as a whole. This too is a form of respect for the human, albeit at a more general level which is perhaps at odds with the current, almost exclusively individualistic interpretation of "respect". Even so, whilst the goals of clinical pathology can express a fine sense of wonder at the human frame, the anonymised mass storage of organs seems to defeat any such sense.

The wider rediscovery of a sense of wonder and celebration of the human form within medicine, alongside the "Faustian itch to explore", will perhaps help prevent our taking the human body for granted, either individually or collectively. We hope that the medical humanities can play their part in this.

\section{References}

1 Porter R. The greatest benefit to mankind. London: Harper Collins, 1997:169.

2 Francis NR, Lewis W. What price dissection? Dissection literally dissected. Medical Humanities 2001;27: (page nos at proof) 3 Boseley S. Alder Hey scandal: survey reveal extent of storage of human tissue. The Guardian 2001 Jan 31: 4. 